\title{
SUMMARY TALK : MULTI-WAVELENGTH SKY SURVEYS
}

\author{
O. LAHAV
}

Institute of Astronomy, Cambridge

\section{Introduction}

An astronomer's career can be viewed in a 3-dimensional space where the (nearly orthogonal) axes are :

- the objects of interest (from planets to the Universe),

- techniques (from instrument design to analytic calculations),

- the wavelength (from the radio to gamma rays).

This interdisciplinary conference brought together experts from different bands of the 'wavelength axis.' It has been an interesting meeting, with a lot of cross-talk, excellent review talks and high-quality posters.

I shall begin by summarizing the highlights (as described in the oral presentations) in different wavelengths, and then discuss the need for statistical techniques, some key astrophysical questions which should be addressed by multi-wavelength (MW) approaches, and the changing sociology of the field.

\section{Across the Spectrum}

For most of the history of mankind our picture of the universe was restricted to the visual band. However, in particular since the Second World War there has been rapid progress in instrumentation and space technology which allows us to have a nearly complete MW picture of astronomical objects and background radiations. Below is a brief summary of what we have heard at the conference, ordered from long to short wavelengths.

\subsection{RADIO}

Being a 'modern' wavelength, many new astronomical phenomena were discovered in the radio. In fact, all Nobel prizes in observational Astronomy 
were given to radio-astronomers: for the discoveries of the Cosmic Microwave Background (CMB) Radiation, the pulsars, and the binary pulsar. At this meeting we have mainly heard about radio extragalactic surveys (Becker, Condon, Disney, Fürst, Sokolov, Wall). Present radio surveys (e.g., 87GB and PMN) have median redshift $\bar{z} \sim 1$, hence providing an unusually deep picture of the universe at early epochs. Recent studies have indicated that radio sources are strongly clustered, suggesting that they reside in high density regions. Several on-going and future surveys in the continuum (FIRST, NVSS, WENSS, UTR) are most promising, although a crucial problem is how to get optical redshifts to these distant radio objects, in order to map their 3-dimensional distribution. The Parkes multibeam survey in $21 \mathrm{~cm}$ promises to give an unbiased view of the neutral hydrogen content in the local universe, with implications for identification of new galaxy populations (e.g., dwarfs and low surface brightness galaxies). Moving to much higher redshifts, radio measurements of temperature fluctuations on the last scattering surface of the CMB by COBE and future experiments (COBRAS/SAMBA, MAP, VSA) reveal the seeds of cosmic structure.

\subsection{INFRARED}

The relatively recent development of detectors in the infrared provides a new window to Galactic and extra-galactic objects (as discussed by Beichman, Oliver, Ruphy). The IRAS data base (in 12, 25, 60 and 100 $\mu$ ) has become a major tool for studies of the Galactic structure and the local universe. In particular, follow-up redshift surveys (IRAS1.2Jy, QDOT, PSCZ) have yielded nearly whole-sky 3-dimensional catalogues for studies of the density and peculiar velocity fields. The new surveys at $2 \mu$ (2MASS, DENIS) are in particular useful for tracing the old 'stable' stellar population in galaxies, and for overcoming the problem of Galactic extinction. Together with ISO, they will give a new picture of stellar populations within the Galaxy and a deeper view of large scale structure.

\subsection{OPTICAL}

This traditional band remains most useful. Photographic surveys (Djorgovski, Morrison, Reid) such as POSS I+II and UK Schmidt still provide the most important wide-angle data bases, e.g., as target lists for redshift surveys. In the old days plates were examined by eye (e.g., Zwicky, Abell, Nilson, Lauberts), but at present the scanning is done by machines (APM, COSMOS, APS, DDS, DPOSS, PMM), with clever software and heroic efforts to calibrate and match plates. It is worth stressing that there is still no whole uniform sky optical catalogue (the nearest to that is a compila- 
tion of UGC, ESO, ESGC, followed up by redshift surveys, e.g., SSRS and ORS).

Existing redshift surveys (e.g., CfA, SSRS, ORS, IRAS, APM, LCRS, CFRS) contain each no more than 30,000 galaxies. A major step forward using multifibre technology will allow us in the near future to produce redshift surveys of millions of galaxies. In particular, two major surveys will probe a median redshift of $\bar{z} \sim 0.1$. The American-Japanese Sloan Digital Sky Survey (SDSS) will yield images in 5 colours for 50 million galaxies, and redshifts for about 1 million galaxies over a quarter of the sky (see reviews by Bahcall, McKay and Szalay). It will be carried out using a dedicated $2.5 \mathrm{~m}$ telescope in New Mexico. A complementary Anglo-Australian survey (discussed by Parker/Taylor), called the 2 degree Field (2dF), will produce redshifts for 250,000 galaxies selected from the APM catalogue. The survey will utilize a new 400 -fibre system on the $4 \mathrm{~m}$ AAT. These surveys will probe scales larger than $\sim 30 h^{-1} \mathrm{Mpc}$. It will also allow better determination of $\Omega$ and bias parameter from redshift distortion. Surveys like $2 \mathrm{dF}$ and SDSS will produce unusually large numbers of galaxy spectra, providing an important probe of the intrinsic galaxy properties, for studying, e.g., the density-morphology relation. Several groups recently devised techniques for automated spectral classification of galaxies utilising, e.g., Principal Component Analysis and Artificial Neural Networks. The SDSS and 2dF projects will also carry out important surveys of quasars (see report by Smith).

We have heard about progress in Astrometry (Egret, Jones, Mignard, Seidelmann). Present and future missions (Hipparcos, Tycho, SIM, GAIA, VSOP) have various applications to a diverse range of topics: e.g., planets, the distance scale and tests of General Relativity. Gravitational lensing (Cook, Schneider) is another innovative area where a major part of the activity is in the optical band. Finally, the impressive pictures of the Hubble Deep Field (reviewed by Dickinson) reveal morphologically disturbed galaxies, probably in the process of formation.

\subsection{UV, EUV}

Th UltraViolet and extreme UV bands (reviewed by Brosch) have been explored by the missions TD1, UIT, GLAZAR and FOCA and future projects include, e.g., TAUVAX, MSX and ARGOS. Here we only point out two important implications of the UV band. The first is that in order to compare local galaxies with the images at high redshift (e.g., from the Hubble Deep Field) one has to image the local galaxies in the UV. The second issue is the relevance of detection of the UV background, which plays an important 
role, e.g., in the evolution of Lyman- $\alpha$ clouds. It is also worth emphasizing the lack of a deep full-sky survey in the UV.

\subsection{X-RAY}

The study of the X-ray band (discussed by Hasinger, Stewart, Trümper) is less than 40 years old, but it opened a new window to high energy phenomena. Satellites like Uhuru, HEAO1, ROSAT, Ginga and ASCA and future missions (ABRIXAS, AXAF, XMM, SRG) probe a wide range of astronomical objects in the X-ray, including comets, binary stars, AGNs, clusters of galaxies and the yet unexplained X-ray Background. We have learned that $50 \%$ of the ROSAT resolved objects are AGNs, $10 \%$ are galaxies and clusters, $35 \%$ stars and $5 \%$ other objects. X-ray clusters are important cosmological probes: the X-ray temperature tells us the depth of the cluster potential well, and hence the total mass, while the X-ray emission indicates a large fraction of baryonic mass, with implications for the density parameter $\Omega$ (see below). Optical follow up observations of the X-ray selected clusters are important in order to get redshifts (so far obtained for 600 clusters).

\section{6. $\gamma$-RAYS}

This is the most 'energetic' band (reviewed by Gehrels and Miller) which in particular became active following the Compton GRO observations. GRO detected Galactic objects, Blazars (which are most probably due to inverse Compton effect in beamed jets), and about $1500 \gamma$-ray bursts. The origin of $\gamma$-ray bursts is still a mystery and a MW identification programme is essential in order to associate them with other astronomical objects at known distances. One current popular theoretical idea is that they are due to merging of 2 neutron stars at cosmological distances. It seems less likely that the $\gamma$-ray bursts reside within the Milky Way. A new innovative project (Milgarno) utilises air showers/Cerenkov radiation to detect very high energy $\gamma$-rays.

\section{Statistical Techniques}

We have heard many talks about cross identification of objects observed at different wavelengths (Bartlett, Becker, Brunner, Hasinger, Helou, Prandoni, Szalay, Wagner, Wall, White). Typical problems are the accuracy of coordinates and overlap of objects. In principle, one should take into account not only the angular proximity, but also the radial distribution of the objects in the different catalogues. For example, the median redshift of the radio survey $87 \mathrm{~GB}$ is $\bar{z} \sim 1$, much deeper than local optical and IRAS 
surveys $(\bar{z} \sim 0.02)$, hence it is not surprising that Condon finds only $1 \%$ of sources in common. One can formulate the cross-identification by taking into account this prior probability for the radial distribution, but it depends to what extent one is prepared to be 'a Bayesian.' It is also possible to calculate the non-zero lag cross-correlation between catalogues. As most objects are known to be clustered, this could yield valuable extra information. An example is cross-correlation of the diffuse X-ray Background with known galaxies.

Other statistical issues discussed at the meeting are selection effects in catalogues (Disney), and the need for innovative methods of classification and pattern recognition (Feigelson, Harwit). Clearly we should keep an open eye on developments of statistical tools in other fields, as many similar problems have already been solved. Special attention was given at this meeting to data bases of astronomical objects and user-friendly software packages (e.g., ADS, CDS, SIMBAD, NED, ALADIN, SkyView) and on how to handle Terabytes of data (Szalay, White). The progress is impressive, and we are all thankful to those who compile the data sets and develop public domain software.

\section{Astrophysical Problems}

Here we outline several key problems in Astrophysics which are best tackles by MW approaches (with a bias of the reviewer towards cosmological problems).

\subsection{GALACTIC STRUCTURE}

The MW measurements and new techniques such as microlensing have renewed interest in the structure and evolution on the Milky Way. Many speakers (Bienaymé, Boulanger, Fürst, Fukui, Gehrels, Larsen, Majewski, Méndez, Parker, Price, Ruphy) discussed unsolved problems related to Galactic structure and dynamics. Here we only list some of them: what is the stellar initial mass function? what is the extent of the thin and thick discs? is the halo lumpy? does the Milky-Way have a bar? how strong is the interaction between the Milky-Way and nearby dwarfs?

\subsection{GALAXIES BEHIND THE ZONE OF AVOIDANCE}

Recent discoveries of galaxies (e.g., the Sagittarius dwarf and Dwingeloo 1) and clusters (e.g., A3627 at the centre of the Great Attractor) illustrated how a combination of eye-balling of plates, $21 \mathrm{~cm}$, infrared and X-ray measurements can be combined to unveil galaxies hidden behind the 'Galactic fog.' Although this topic was not directly discussed at this meeting, it is 
clear that collaboration of Galactic and extragalactic astronomers on this topic can benefit both groups. There is also a great need for a better extinction map of the Galaxy (e.g., by combining optical, UV, HI, IRAS and COBE maps). We were reminded that there is substructure in the ISM even at the north and south Galactic poles.

\subsection{GALAXY FORMATION}

A MW approach is required to produce the 'H-R diagram' of galaxies. Rather than talking just about a luminosity function in one colour, one should consider a multivariate function which includes luminosities in various spectral bands and dynamical properties of galaxies. Deeper galaxy images such as the HDF hold the key to issues of galaxy formation and evolution (e.g., Dickinson's review), but to make sense of the high redshift measurements it is crucial to improve our knowledge of galaxy properties in the local universe. Another constraint on galaxy formation can be obtained from the 'maximum' redshift of QSOs (Osmer, Padovani).

\subsection{LARGE SCALE STRUCTURE \& COSMOLOGY}

Recent work on understanding clusters of galaxies is a good example of MW measurements. Optical, X-ray, CMB (for the Sunyaev-Zeldovich effect) and gravitational lensing are combined to quantify the extent and matter content of clusters (discussed by Bahcall, Trümper, Schneider). For example, the high fraction of baryons in clusters suggests that $\Omega \approx 0.2$, in conflict with the popular $\Omega=1$ value. The derivation of $\Omega$ from comparison of density and velocity fields and from redshift distortion is affected by 'biasing,' i.e., the way galaxies trace the underlying mass distribution. Galaxies observed at different wavelengths (e.g., optical and IRAS) have different clustering properties, hence a different 'bias parameter.' This conceptual issue will remain crucial in the interpretation of the 2dF and SDSS surveys, and in connecting the power-spectrum of density fluctuations from galaxy surveys and the CMB.

MW studies are also important for understanding the background radiations. Most of the background energy is in the CMB $\left(0.25 \mathrm{eV} \mathrm{cm}^{-3}\right)$, compared with, e.g., the XRB $\left(5 \times 10^{-5} \mathrm{eV} \mathrm{cm} \mathrm{cm}^{-3}\right)$. Only upper limits (from direct measurements) are available for the optical and UV backgrounds. An interesting example of MW approach is how the hot intergalactic medium model for the XRB was ruled out by the lack of distortion of the blackbody CMB spectrum. It is also important to detect the dipole in various background radiations, to confirm that the CMB dipole is due to motion. Sub-degree measurements of fluctuations in the CMB by new experiments (COBRAS/SAMBA, MAP, VSA) will provide a new insight into the nature 
of the dark matter, and will yield estimation (in a model-dependent way) of the cosmological parameters (e.g., $\Omega$ and $\left.H_{0}\right)$ to within a few percent.

\section{Changing Sociology}

We have heard at this meeting about future big telescopes, big collaborations and big data bases. Several speakers made the remarks "it takes at least 2-3 times the most pessimistic estimate to begin/complete/analyse a survey" and "to make a big impact, a new survey must be 10-1000 times better in sensitivity/resolution/number of objects." This indicates that it is becoming more and more challenging to make an impact by conducting big surveys. It also raises some questions about the changing sociology of doing research in astronomy: what will be the individual's contribution in a big collaboration (cf. particle physics experiments)? what skills should be acquired by the next generation of astronomers? Will the increase in projects and data sets be followed by more jobs for young astronomers? How would the community deal with public domain data (e.g., HDF)? and how to communicate the knowledge resulting from the surveys to the tax payer? While focusing on the technological aspects of the MW surveys, the human aspects should not be forgotten.

\section{Acknowledgements}

Finally, many thanks to the scientific and local organizing committees, in particular to Barry Lasker and Marc Postman, for organizing this interesting and stimulating meeting. 